\title{
A fuzzy clustering approach to a demand response model
}

\author{
R. Pereira ${ }^{\text {a,c }}$, A. Fagundes ${ }^{\mathrm{c}}$, R. Melício ${ }^{\mathrm{a}, \mathrm{b}}$, V.M.F. Mendes ${ }^{\mathrm{a}, \mathrm{c}}$, \\ J. Figueiredo ${ }^{\mathrm{a}, \mathrm{b}}$, J. Martins ${ }^{\mathrm{a}}$, J.C. Quadrado ${ }^{\mathrm{c}}$ \\ (rpereira@ deea.isel.ipl.pt; fagundesready@gmail.com; ruimelicio@gmail.com; vfmendes@deea.isel.ipl.pt; jfig@uevora.pt; \\ jf.martins@fct.unl.pt; jcquadrado@gmail.com) \\ ${ }^{a}$ Department of Physics, Universidade de Évora, Largo dos Colegiais 2, 7004-516 Évora, Portugal \\ ' IDMEC/LAETA, Instituto Superior Técnico, Universidade de Lisboa, Lisbon, Portugal \\ ${ }^{\mathrm{c}}$ Department of Electrical Engineering and Automation, Instituto Superior de Engenharia de Lisboa, R. Conselheiro Emídio \\ Navarro, 1959-007 Lisbon, Portugal \\ * Corresponding author, Tel: +35121831 7285, Fax: +35121831 7271. \\ E-mail adress: rpereira@deea.isel.ipl.pt (R. Pereira).
}

\begin{abstract}
This paper proposes a novel demand response model using a fuzzy subtractive cluster approach. The model development provides support to domestic consumer decisions on controllable loads management, considering consumers' consumption needs and the appropriate load shape or rescheduling in order to achieve possible economic benefits. The model based on fuzzy subtractive clustering method considers clusters of domestic consumption covering an adequate consumption range. Analysis of different scenarios is presented considering available electric power and electric energy prices. Simulation results are presented and conclusions of the proposed demand response model are discussed.
\end{abstract}

Highlights

- Existing demand response models are summarized;

- A new demand response model is developed with fuzzy subtractive clustering techniques;

- The developed demand response model provides the management of controllable loads to consumer;

- The proposed model efficiently limits the consumption below the available power;

- Load shedding and valley filling can be achieved with the proposed demand response model;

Keywords: Demand response; smart grid; fuzzy clustering; load management.

\section{Introduction}

Smart grid concept is at the present time the main motivation for future electricity systems development [1] contributing to an electricity systems convergence, providing further opportunities to distributed generation integration (mainly renewable energy) and allowing consumers to assume proactive actions in demand side management [2]. The success of smart grids depends greatly on the motivation of consumers to play an active role in grid management. Therefore, the consumer's engagement in smart grid first stage initiatives is considered crucial to avoid failing risks [1]. In smart grid context, demand response (DR) is an essential demand side management action for consumers [3]. In fact, the behavior management is of primer importance, namely in what the power consumption shaping is concerned, 